\title{
Hugo Hadwiger's influence on geometric dissections with special properties
}

\author{
Greg N. Frederickson
}

On the occasion of the Swiss Mathematical Society's centennial, it is both an honor and a pleasure to contribute an article to this special edition of the Elemente der Mathematik on a mathematical subject from the work of Hugo Hadwiger. Professor Hadwiger was a prolific and influential Swiss mathematician known for his work in geometry, combinatorics, and cryptography [2]. Here we shall note how his ideas in dissection geometry have been extended into the present. The focus will proceed from his article, "Zerlegungsgleichheit ebener Polygone" [13], co-authored with Paul Glur, which appears to be the most-referenced of his articles on dissection. In the almost sixty years since this article appeared, several popular books on geometric dissections $[4,5,6,15]$ have been published, a superb website [17] has been created, and an early manuscript [12] has been recovered.

A dissection possesses translation with no rotation, or is translational, if the pieces can be moved from one figure to the other without rotating them. It is not always possible to find dissections between two figures that possess this property, no matter how the figures are oriented with respect to each other. Hadwiger and Glur gave a simple characterization of which figures can have a dissection that has this property. For each figure, walk around its boundary in a clockwise direction, and identify the slopes of each line segment in the boundary. Each line segment has a slope and a direction, either to the right or to the left, over which it is walked. (For vertical line segments, replace right and left by up and down.) For each figure, and for each slope of line segments in that figure, add the lengths of the line segments walked to the right and subtract from this sum the lengths of line segments walked to the left. There will be a translational dissection if and only if for every slope, the sum for one figure equals the sum for the other.

An example is the pair consisting of a square and an equilateral triangle in Fig. 1(a). There is no translational dissection of a triangle to a square, because the sums of opposite pairs of sides in the square sum to zero, while each of the three sides of the triangle contributes a nonzero value that is not offset by any other side. The dissection by Harry Lindgren [15] of four pentagons to one, shown in Fig. 1(b), is an example that is translational. With one of the small pentagons oriented inversely to the other three, the sums of the side lengths for all five slopes are the same for the four small pentagons, as for the large one. 

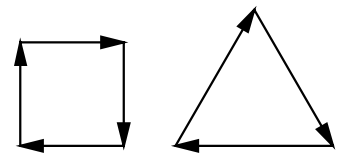

Fig. 1 (a) Square versus triangle

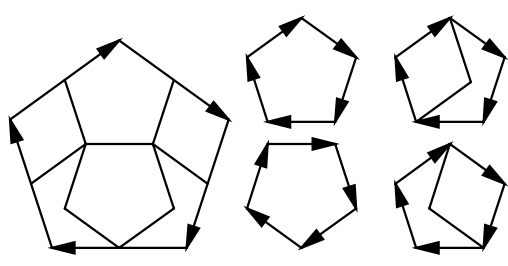

(b) Four pentagons to one

When a pair of figures satisfies Hadwiger and Glur's requirement, it may not be possible to find a dissection that is translational and also uses as few pieces as some nontranslational dissection. An example by Lindgren [15] is the dissection of a Latin Cross and the $\{12 / 5\}$ star in Fig. 2. The sum for each slope in the Latin Cross is zero, and similarly for the $\{12 / 5\}$ star, so that there is a translational dissection. However it seems unlikely that there will be any translational dissection with as few as seven pieces.
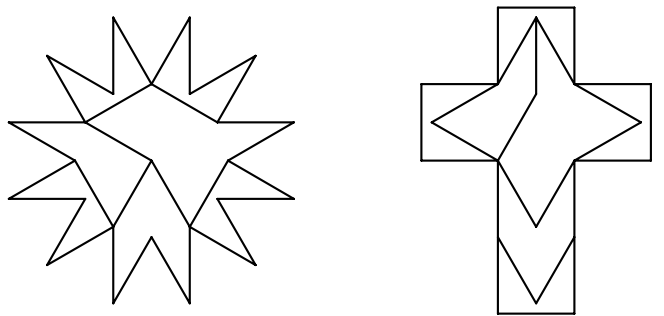

Fig. 2 Non-translational dissection of a $\{12 / 5\}$ star to a Latin Cross

Further simple properties. Another property of a geometric dissection is whether no pieces need be turned over when pieces are moved from one figure to the other. Harry Lindgren [15] identified some dissection problems for which solutions with fewer pieces are known if pieces are allowed to be turned over. An example of such a dissection is the 6-piece dissection of a hexagram to a hexagon from [4], shown in Fig. 3(a), in which the two nonequilateral triangles need to be turned over.

A further property is whether none of the cuts are curved, i.e., all are polygonal. Gavin Theobald [17] discovered how to modify the hexagram-to-hexagon dissection in Fig. 3(a) so that no pieces are turned over, as shown in Fig. 3(b). He enlarged the two nonequilateral
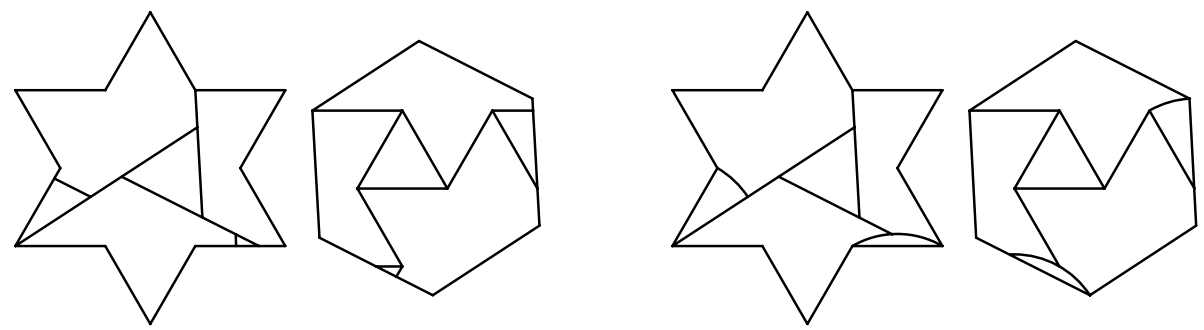

Fig. 3 Hexagram to hexagon: (a) with turnover, (b) with no turnover 
triangles by introducing curved cuts, so that each resulting piece has mirror symmetry. One triangular piece becomes a "pie slice," while the other is now the portion of a circle cut off by a chord. The circular cuts have radius equal to the length of the edge of the hexagram. This appears to be the first essential use of curved cuts in a dissection of figures that do not have curved boundaries.

Returning to translational dissections, consider a translational dissection cut out of a piece of wood so that all pieces from one figure have the wood grain running in the same direction. If the pieces are assembled into the other figure, then all pieces will once again have the wood grain running in the same direction. Thus every translational dissection is grainpreserving. Besides wood, another material that has a similar property is polarized glass.
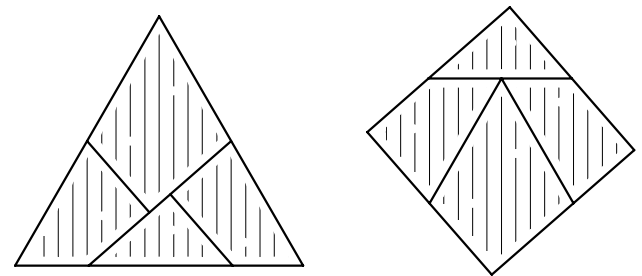

Fig. 4 Grain-preserving dissection of an equilateral triangle to a square

There are some dissections that are not translational but are nonetheless grain-preserving. In fact, any dissection that uses just the two motions of translation and rotation by $180^{\circ}$ will be grain preserving. Thus the 4-piece dissection of an equilateral triangle to a square in Fig. 4 is grain-preserving. In fact, there is a grain-preserving dissection between any two polygonal figures of equal area. Merely cut one polygon into triangles, and then transform each triangle into a parallelogram using one cut, one rotation by $180^{\circ}$, and then one translation. There is a translational dissection that takes the parallelogram to a rectangle of suitable length, and then the rectangles for each of the triangles can be stacked. Working from the other polygon, a similar transformation can be effected. The two stacks can then be superposed to produce the complete dissection.

Swing-hingeability. A remarkable property evidenced by some dissections is hingeability. In his book, The Canterbury Puzzles, Henry Ernest Dudeney introduced a hinged version of the apparently unique 4-piece dissection of an equilateral triangle to a square. The dissection is shown in Fig. 5 with a hinging of its pieces. It is swing-hingeable because the pieces can be linked together with swing hinges, so that when they are swung one way on the hinges, the pieces form one figure, and when swung around the other way they form the other figure.

Several natural questions arise regarding swing-hinged dissections:

1. Given two polygonal figures of equal area, does a swing-hinged dissection of these figures exist?

2. If so, how does one find such a swing-hinged dissection?

3. Given a dissection, a swing-hinging, and two swing-hinged configurations, is there a sequence of motions that takes the dissection from one swing-hinged configuration to the other? 

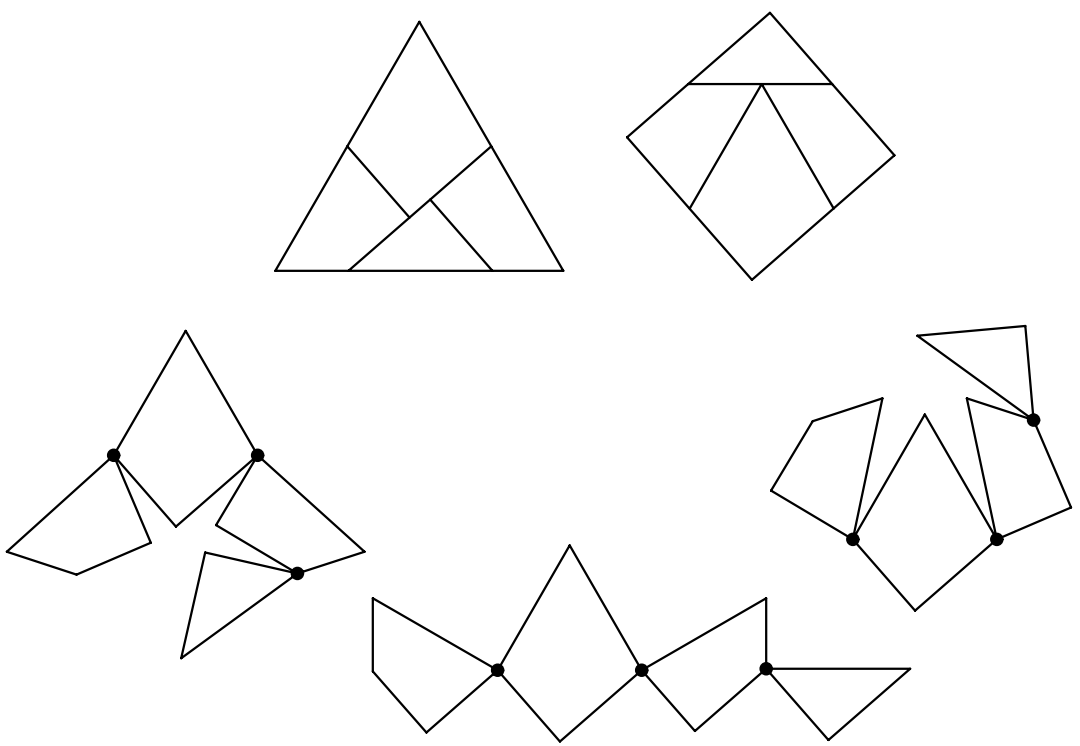

Fig. 5 Swing-hinged dissection of an equilateral triangle to a square

My book on hinged dissections [5] approached the first two of these questions by identifying many new pairs of polygonal figures that had hinged dissections, and by identifying a number of specific techniques for producing such dissections. It was then proved in [14] that the third question is PSPACE-hard [16], even when the configuration shapes are convex polygons. More recently, the first two questions have been resolved in [1] by showing that there exists a hinged dissection between every pair of polygons of equal area and by giving a general procedure to construct such a hinged dissection. The solution is ingenious but leads to complex dissections.
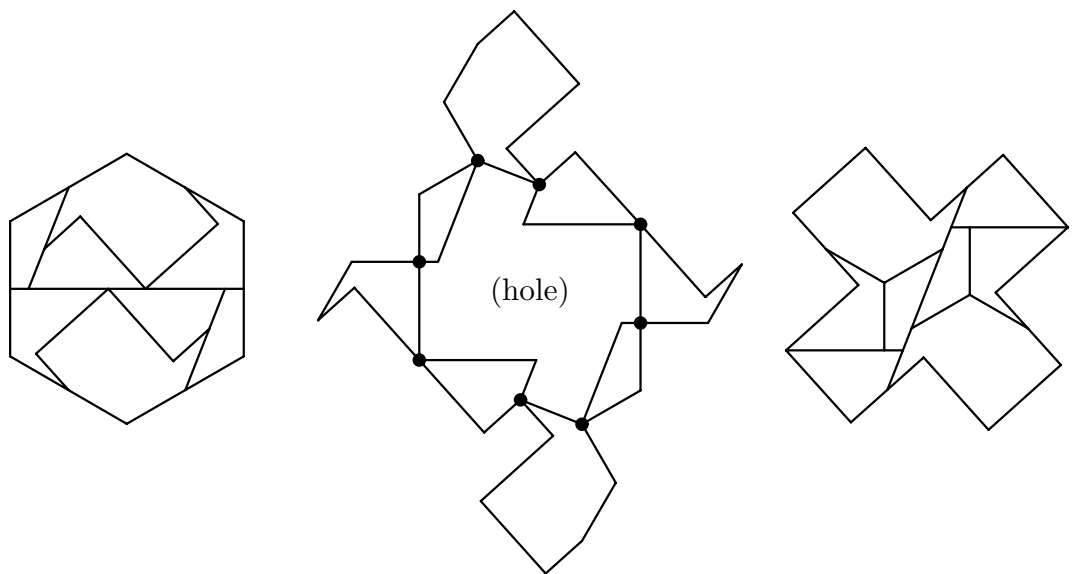

Fig. 6 Swing-hinged dissection of a Greek Cross to a regular hexagon 
As previously shown, the swing-hinged dissection of a triangle to a square in Fig. 5 is grain-preserving. A different swing-hinged dissection of an equilateral triangle to a square, and one that is suitable as the top of a card table and that can be supported by one pedestal leg, is presented in [9]. The top of that table is also grain-preserving.

A hinged assemblage is cyclicly hinged if a subset of pieces and hinges forms a cycle, and each hinge is shared by just two pieces. An example of a cyclicly hinged dissection is the dissection of a Greek Cross to a hexagon in Fig. 6. Note that the cuts in both the Greek Cross and the hexagon have 2-fold rotational symmetry. To swing open the hexagon, keep the three pieces on the lower right of the hexagon together, and similarly for the three pieces on the upper left. The hole opens in the shape of a parallelogram.

Twist-hingeability. A twist hinge is a hinge that uses a point of rotation on the interior of an edge shared by two pieces [6]. With a twist hinge, one piece is flipped over relative to the other, using rotation by $180^{\circ}$ through the third dimension. Pieces A and B (with exaggerated thickness) are twist-hinged together in Fig. 7.

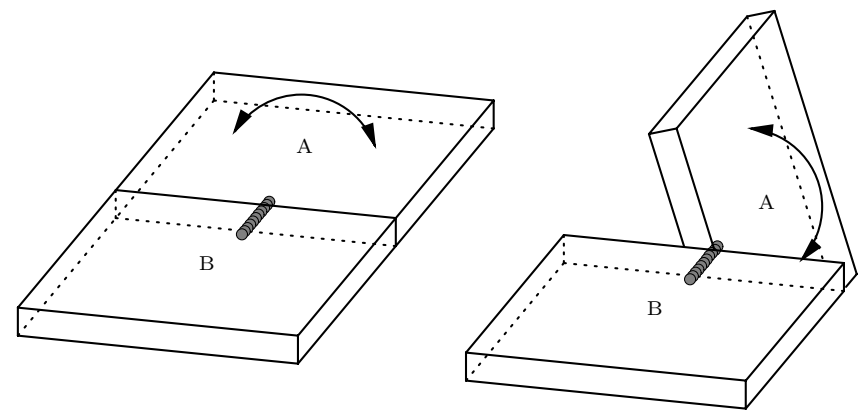

Fig. 7 A twist hinge between pieces $\mathrm{A}$ and $\mathrm{B}$

It is often possible to convert a swing-hinged dissection into one that is twist-hinged. The technique requires that each swing hinge connects two pieces and that the hinged pieces are "hinge-snug." Two pieces connected by a swing hinge are hinge-snug if they are adjacent along different parts of the boundaries in each of the target figures, and each such part (a line segment) has one endpoint at the hinge. Then the swing hinge can be replaced by a new piece and two twist hinges. The new piece is the union of two isosceles triangles, one cut from each piece attached to the swing hinge.

With this technique, it is possible to derive a 7-piece twist-hinged dissection of an equilateral triangle to a square by converting the 4-piece swing-hinged dissection in Fig. 5. Fig. 8 shows the cuts in the triangle and in the square, plus dashed edges to indicate the bases of the isosceles triangles adjacent to each hinge point. In Fig. 9, the pairs of isosceles triangles are merged together, and the twist hinges are indicated by small circles. Those pieces that are flipped an odd number of times when converting from one figure to the other are labeled with an asterisk or a star.

There is a family of twist-hinged dissections from [5] that is beautifully symmetric. For any integer $p>2$, there is a $(2 p+1)$-piece twist-hingeable dissection of a $\{2 p\}$ (a regular polygon of $2 p$ sides) to a $\{p\}$ (a regular $p$-polygon). These dissections exhibit $p$-fold rotational symmetry. A twist-hinged dissection of a hexagon to a triangle is shown in Fig. 10. 

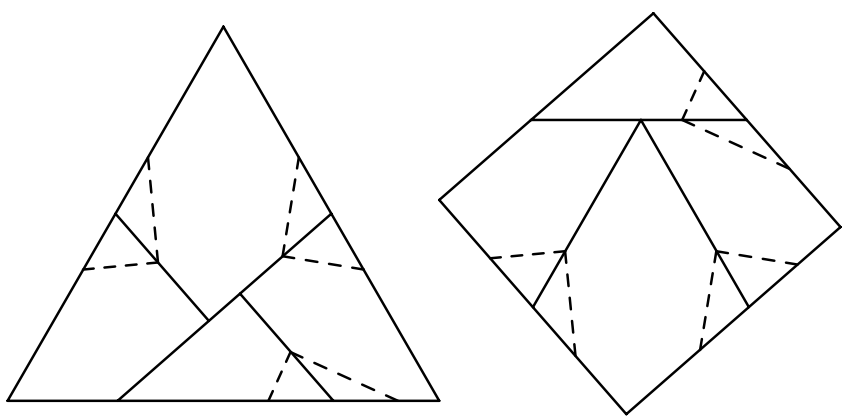

Fig. 8 Adding isosceles triangles to the triangle-to-square dissection
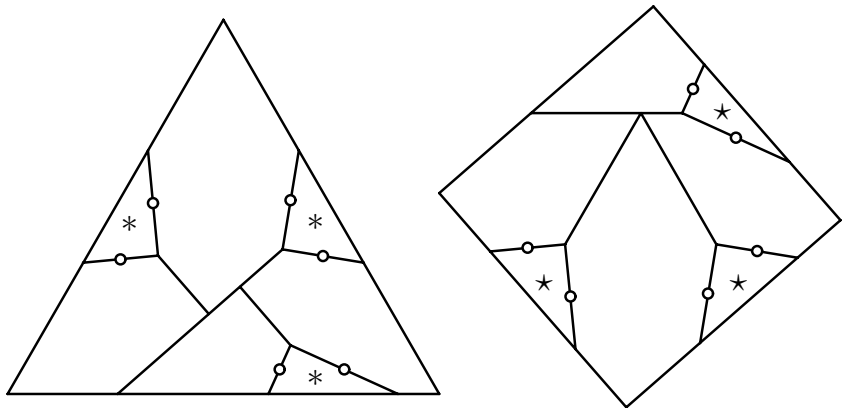

Fig. 9 Twist-hinged dissection of an equilateral triangle to a square

Produce the dissection as follows. Overlay the $\{2 p\}$ and the $\{p\}$ so that their centers coincide and every side of the $\{p\}$ bisects a side of the $\{2 p\}$. For each vertex A of the $\{p\}$, draw a line segment to the nearest vertex B of the $\{2 p\}$. For each such vertex B of the $\{2 p\}$, identify the vertex $\mathrm{C}$ of the $\{2 p\}$ such that a side of the $\{p\}$ bisects side BC. Draw
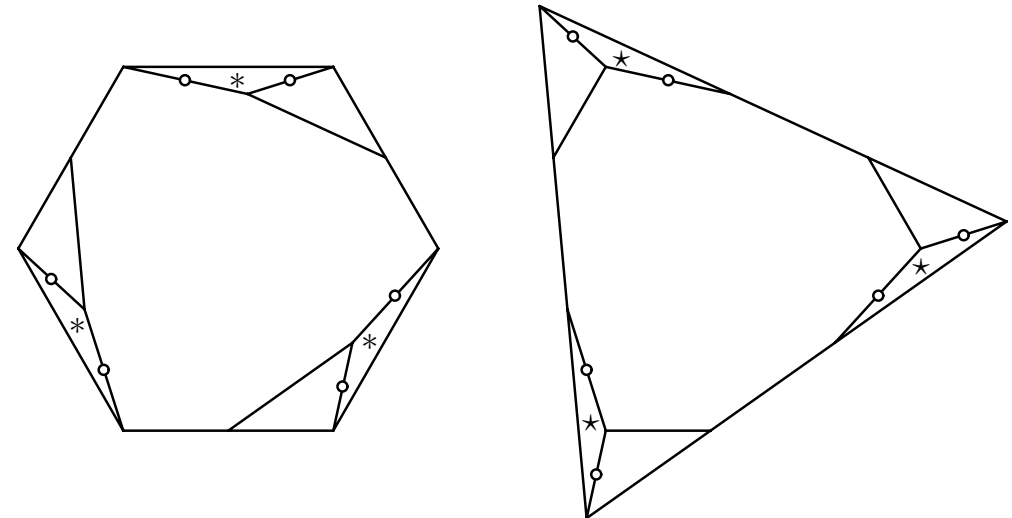

Fig. 10 Twist-hinged dissection of a hexagon to a triangle 
a line segment from $\mathrm{C}$ to the nearest side of the $\{p\}$, with the line segment parallel to the line segment $\mathrm{AB}$. From the other endpoint $\mathrm{D}$ of this line segment, draw a line segment to the next vertex $\mathrm{B}^{\prime}$ of the $\{2 p\}$ after $\mathrm{C}$. In addition to the sides of $\{2 p\}$ and $\{p\}$, these line segments identify the appropriate cuts to make.

Special cases of twist-hinged dissections are explored further in [7, 8]. One challenging example is of a heptagon to a square, which is derived from a 7-piece unhingeable dissection due to Gavin Theobald [4, Fig. 11.30]. Although there are just two positions where swing hinges can be placed, it is still possible to convert this dissection to a 10-piece twisthinged dissection. The conversion involves cutting and annexing an isosceles triangle and also cutting an isosceles trapezoid. After converting the two swing hinges, one more piece can be connected by a twist hinge to come along for the ride. The resulting dissection is shown in Fig. 11.
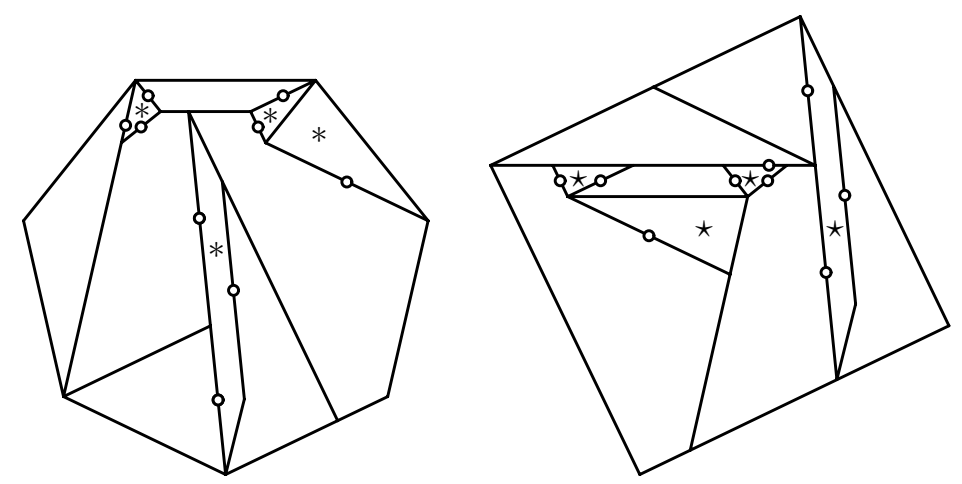

Fig. 11 Twist-hinged dissection of a regular heptagon to a square

Foldability. A third type of hinge is a piano hinge, which allows two flat pieces to fold together or apart [6]. (The term piano hinge comes from the long hinge that attaches the lid of a grand piano to the box containing the strings.) Let each polygon be a shallow prism separated into two levels which can be further dissected into flat pieces. A piano-hinge connects two flat pieces that are side-by-side on the same level and allows one piece to flip on top of the other.

If each level were truly two-dimensional, i.e., with no thickness, then two pieces connected by a piano hinge would share points in common when folding them against each other in three-dimensional space. This problem can be avoided by assuming that each piece is the union of one or more prisms of thickness $\epsilon$, for some small $\epsilon>0$. The axis of rotation of a piano hinge will coincide with an edge on the shared boundary of the two pieces connected by the hinge. The left side of Fig. 12 displays schematically two such prisms hinged together, while the right side shows that it is easy to rotate piece A by $180^{\circ}$ to bring it to lie on top of piece B.

A piano-hinged dissection is cyclicly hinged if it is possible to remove one of the hinges without disconnecting any of the pieces from the others. This surplus of hinges forces various pieces to move in a coordinated fashion. There are several ways to produce a cyclic hinging. For ease in characterization, imagine that $\epsilon$ has shrunk to zero. The first 

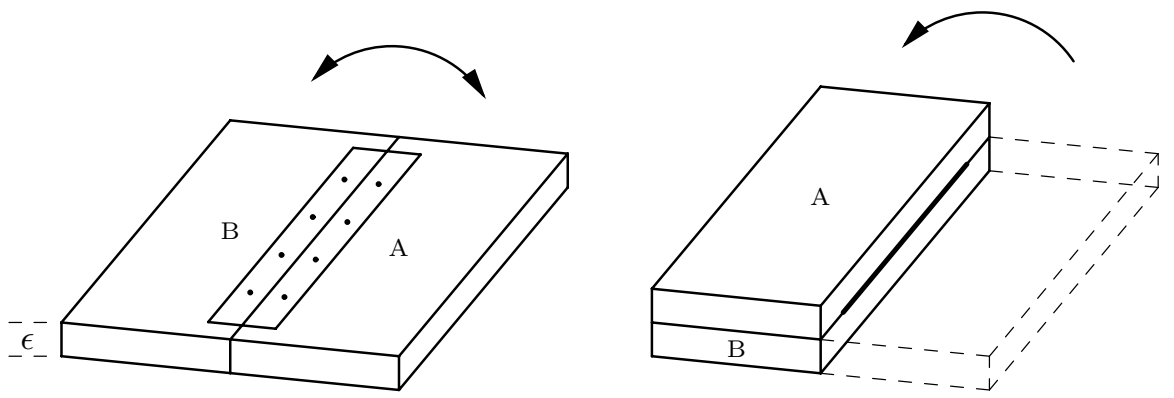

Fig. 12 Folding, from A next to B, to A on top of B ...

type of hinging, a vertex-cyclic hinging, has four or more pieces that touch at a common vertex, and each piece is hinged with its predecessor and its successor on the cycle.

Let $2 \sigma$ be the sum of the angles that meet at the vertex. If $\sigma<180^{\circ}$, then the vertex-cyclic hinging is a cap-cyclic hinging. Pieces D, E, F, and G are cap-cyclicly hinged in Fig. 16. Here a dotted line is used to show a piano-hinge between two pieces on the same level. A pair of pieces, one on top of another, that are hinged together are designated by a row of dots next to their shared boundary on each level.

If $\sigma=180^{\circ}$, then the vertex-cyclic hinging is a flat-cyclic hinging. Since this cyclic hinging is straightforward, we omit further mention of it here.

If $\sigma>180^{\circ}$, then the vertex-cyclic hinging is a saddle-cyclic hinging. This cycle will be at a concave vertex in the boundary of each target figure of the dissection. Its name derives from the shape of the surface as it moves from one configuration to the other. The assemblage will open from a concave angle to a surface with a saddle point and then close to a concave angle on the other side of the surface. At a saddle point (of a surface shaped
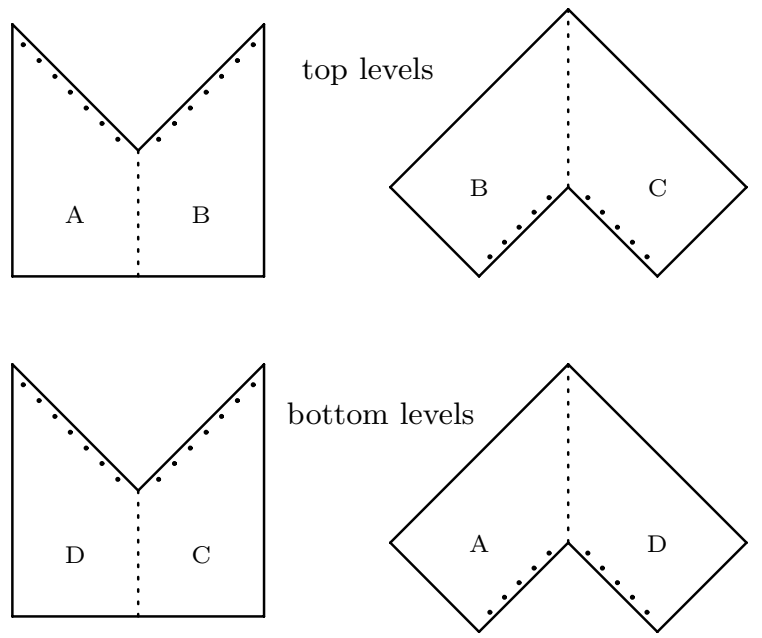

Fig. 13 Saddle-cyclicly hinged dissection of a mitre to a gnomon 

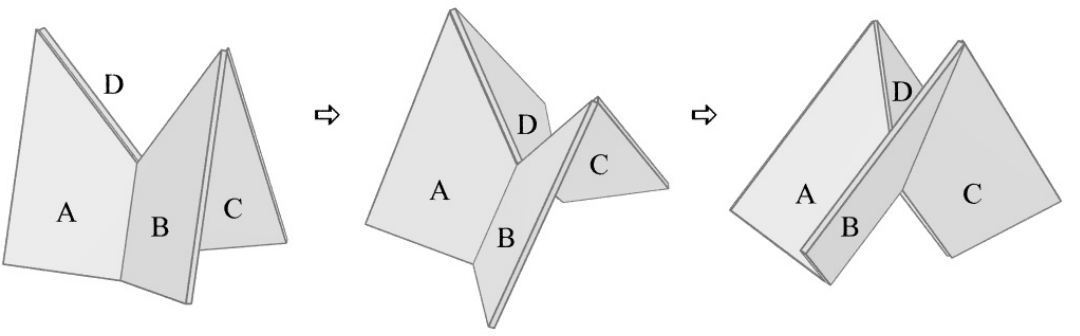

Fig. 14 Saddle-cyclicly folding a mitre to a gnomon

like a horse's saddle), one cross-section of the surface attains a maximum while another cross-section of the surface attains a minimum.

An example is the piano-hinged dissection of a mitre to a gnomon in Fig. 13. A mitre is what remains from a square after cutting away an isosceles right triangle whose hypotenuse coincides with a side of the square. A gnomon is what remains from a square after cutting away a square of one fourth the area from a corner of the larger square. Both the mitre and the gnomon have an angle of $\sigma=270^{\circ}$. On each level cut the figure in two, splitting these large angles and creating four pieces that meet at angles totaling $540^{\circ}$. Fig. 14 shows how to fold from the mitre to the gnomon.

Stack-foldability. A natural extension of a piano-hinged dissection is a dissection in which the heights of the two figures are not identical: Given two figures of equal volume, where one is $p$ levels thick and the other is $q \neq p$ levels thick, and $p$ and $q$ are whole numbers, is it possible to create just one assemblage that will fold to form each of the figures in turn? In such a case, a natural goal is to find an assemblage with as few pieces as possible, and beyond that to use cyclic hingings when possible [6].

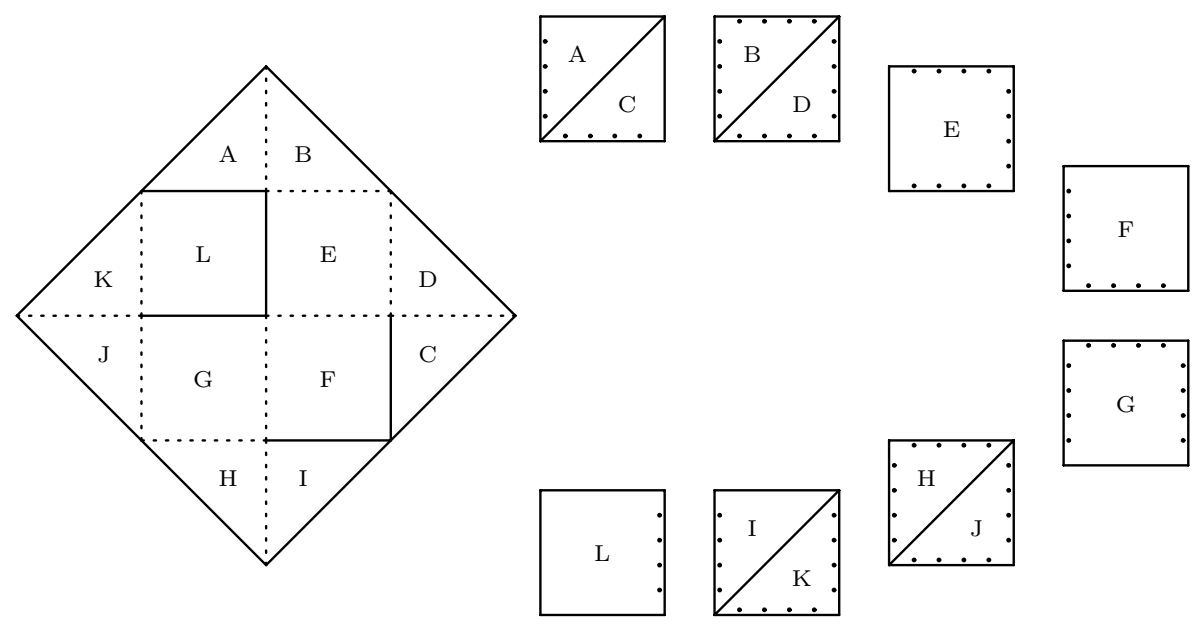

Fig. 15 Stack-folding dissection of a 1-level square to an 8-level square 
An example is a folding dissection of a 1-level square to an 8-level square [11]. It is possible to adapt a simple 12-piece unhinged dissection by Ernest Freese [12]. In Fig. 15, the levels of the 8-level square snake around from the top middle of the figure to the bottom middle. The four pairs of isosceles right triangles from the 1-level square are stacked in the 8-level square so that four of these triangles (pieces A, B, C, and D) land above piece E, and four more of them (pieces H, I, J, and K) land between pieces G and L. Note that pieces $\mathrm{J}$ and $\mathrm{K}$ share just a single piano hinge, not two. The second hinge for piece $\mathrm{J}$ connects to piece $\mathrm{G}$, and the second hinge for piece $\mathrm{K}$ connects to piece $\mathrm{L}$.

A final example is a dissection of equilateral triangular prisms, from [10]. Ernest Freese identified a simple 12-piece unhingeable dissection of four triangles to three. It can be adapted into a 10-piece stack-folding dissection of a 4-level triangle to a 3-level triangle, as in Fig. 16. The four levels of the 4-level triangle appear left to right on the top row of
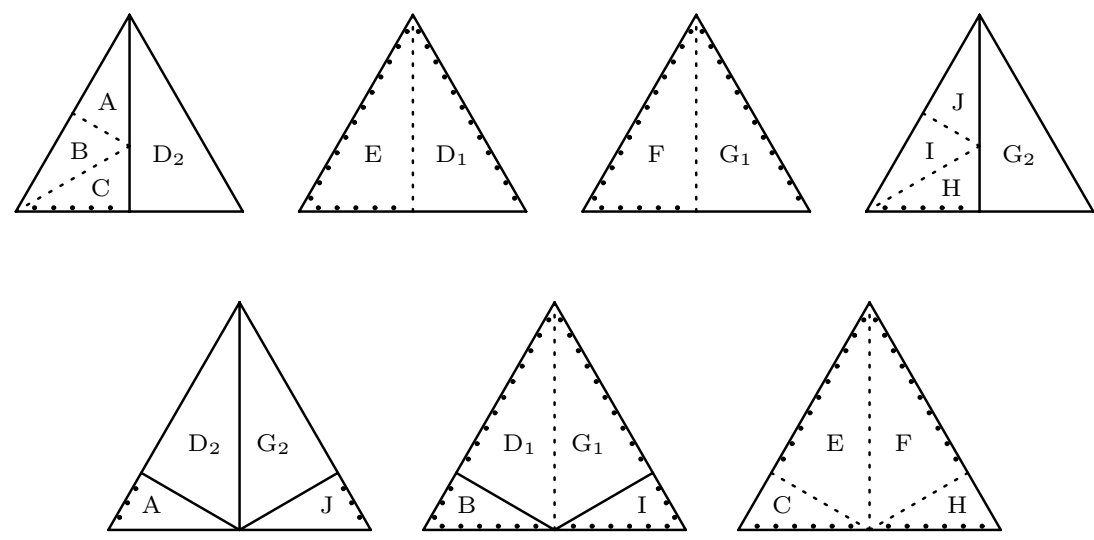

Fig. 16 Stack-folding dissection of a 4-level triangle to a 3-level triangle

Fig. 16, while the three levels of the 3-level triangle appear left to right on the bottom row. Freese cut three of the four congruent triangles into two mirror-image right triangles, and cut the fourth triangle into six smaller right triangles. Here the six small right triangles are grouped together into two groups of three, with each group filling half a level in the 4-level triangle and extending through all three levels in the 3-level triangle. Note that pieces D and $G$ are each two levels high, and that pieces D, E, F, and G are cap-cyclicly hinged.

Conclusion. Many intriguing properties of geometric dissections have been explored in the years since Hugo Hadwiger and Paul Glur published their foundational work on translational dissections. The properties have included restrictive rotations, primarily based on a variety of hinges attached to the dissection pieces. It is hoped that the work of Hadwiger and Glur will continue to inspire mathematicians well into the future.

Animations for a selection of dissections in this article are displayed on the webpage:

http: //www. cs. purdue. edu/homes/gnf/book2/beyondH . html 


\section{References}

[1] Abbott, T.G.; Abel, Z.; Charlton, D.; Demaine, E.D.; Demaine, M.L.; Kominers, S.D.: Hinged dissections exist. In: Proceedings of the 24th Annual ACM Symposium on Computational Geometry, College Park, Maryland 2008, 110-119.

[2] Debrunner, H.E.; Mani, P.; Meier, C.; Rätz, J.; Streit, F.: Professor Dr. Hugo Hadwiger, 1908-1981. Elem. Math. 37 (1982) 3, 65-78.

[3] Dudeney, H.E.: The Canterbury Puzzles and Other Curious Problems. W. Heinemann, London 1907.

[4] Frederickson, G.N.: Dissections Plane \& Fancy. Cambridge University Press, New York 1997.

[5] Frederickson, G.N.: Hinged Dissections: Swinging and Twisting. Cambridge University Press, New York 2002.

[6] Frederickson, G.N.: Piano-hinged Dissections: Time to Fold. A K Peters Ltd, Wellesley, Massachusetts 2006.

[7] Frederickson, G.N.: The heptagon to the square, and other wild twists. Math. Intelligencer 29 (2007) 4 , 23-33.

[8] Frederickson, G.N.: Unexpected twists in geometric dissections. Graphs Combin. 23[Suppl] (2007), 245258

[9] Frederickson, G.N.: Designing a table both swinging and stable. College Math. J. 39 (2008) 4, $258-266$.

[10] Frederickson, G.N.: Dissecting and folding stacks whose cross-sections are equilateral triangles. Manuscript, 2009.

[11] Frederickson, G.N.: Dissecting and folding stacked geometric figures. Manuscript, April 2010.

[12] Freese, E.I.: Geometric transformations. A graphic record of explorations and discoveries in the diversional domain of Dissective Geometry. Unpublished, 1957.

[13] Hadwiger, H.; Glur, P.: Zerlegungsgleichheit ebener Polygone. Elem. Math. 6 (1951), 97-106.

[14] Hearn, R.A.; Demaine, E.D.; Frederickson, G.N.: Hinged dissection of polygons is hard. In: Proceedings of the 15th Canadian Conf. on Computational Geometry, Halifax, Canada 2003, 98-102.

[15] Lindgren, H.: Geometric Dissections. D. Van Nostrand Company, Princeton, New Jersey 1964.

[16] Sipser, M.: Introduction to the Theory of Computation. Thomson Course Technology, Boston, $2^{\text {nd }}$ ed., 2006. See Section 8.3.

[17] Theobald, G.: Geometric dissections. Collection of webpages (http://home.btconnect.com/ GavinTheobald/Index.html), 2004.

Greg N. Frederickson

Department of Computer Science

Purdue University

West Lafayette, IN 47907, USA

e-mail: gnf@cs.purdue.edu 